\title{
Circulating dehydroepiandrosterone sulfate level and cardiovascular or all-cause mortality in the elderly population: a meta-analysis
}

\author{
Rui $\mathrm{Li}^{1}$, Lihua $\mathrm{E}^{2}$, Nashunbayaer $\mathrm{Zha}^{3}$ \\ ${ }^{1}$ Department of General Practice, ${ }^{2}$ Department of Stomatology, ${ }^{3}$ Department of Thoracic Surgery, The Affiliated Hospital of Inner Mongolia \\ Medical University, Hohhot, China \\ Contributions: (I) Conception and design: N Zha; (II) Administrative support: N Zha; (III) Provision of study materials or patients: R Li; (IV) \\ Collection and assembly of data: R Li, L E; (V) Data analysis and interpretation: R Li, L E; (VI) Manuscript writing: All authors; (VII) Final approval \\ of manuscript: All authors. \\ Correspondence to: Doctor. Nashunbayaer Zha (ORCID: 0000-0002-9449-9871). Department of Thoracic Surgery, The Affiliated Hospital of Inner \\ Mongolia Medical University, No.1 Tongdao North Road, Hohhot, 010050, China. Email: nashun1937@sina.com.
}

\begin{abstract}
Studies have yielded contradictory results concerning the association between dehydroepiandrosterone sulfate (DHEAS) and mortality in the elderly population. This meta-analysis aimed to evaluate the association of low serum DHEAS level with cardiovascular or all-cause mortality in the elderly population. A comprehensive literature search was conducted in PubMed and Embase databases up to 4 February, 2019. Longitudinal observational studies reporting multivariate adjusted risk ratio (RR) and corresponding $95 \%$ confidence intervals (CI) for cardiovascular or all-cause mortality with respect to baseline low DHEAS level were included. Both fixed-effect and random effect model were used to pool the overall risk estimate. Methodological quality of the included studies was evaluated using a 9-point Newcastle-Ottawa Scale. Six prospective studies enrolling 6,744 individuals were identified. Five studies were graded as high methodological quality. When compared the lowest to the reference higher circulating DHEAS level, the pooled RR of all-cause and cardiovascular mortality was 1.46 (95\% CI: 1.25-1.70) and 1.49 (95\% CI: 1.11-1.99), respectively. Subgroup analysis indicated that the association of low DHEAS level with all-cause mortality risk was only found in men (RR 1.41;95\% CI: 1.18-1.69) but not in women (RR 1.72; 95\% CI: 0.99-2.99). This meta-analysis provides evidence that low circulating DHEAS level is associated with increased risk all-cause mortality in the elderly population.
\end{abstract}

Keywords: Dehydroepiandrosterone sulfate (DHEAS); cardiovascular mortality; all-cause mortality; meta-analysis

Submitted Feb 17, 2020. Accepted for publication Aug 07, 2020.

doi: 10.21037/apm-20-441

View this article at: http://dx.doi.org/10.21037/apm-20-441

\section{Introduction}

Dehydroepiandrosterone sulfate (DHEAS), also known as androstenolone sulfate, is an endogenous androstane steroid that is present in both male and female. Almost all DHEAS is synthesized in the adrenal cortex and only minimal level of DHEAS is produced by the testes (1). DHEAS can be converted into testosterone, androstenedione, and estrogen (2). Men have higher circulating DHEAS level than women (3). The production of DHEAS peaks in young adults and decreases with advancing age in both genders (4). Agerelated decline in DHEAS level has been implicated in the aging process (5), which means DHEAS may play an important role in the longevity (6).

Low level of serum/plasma DHEAS has been linked to impaired survival prognosis in the patients with cardiovascular disease $(7,8)$, hemodialysis (9), and chronic obstructive pulmonary disease (10). There are several possible explanations for low circulating level of DHEAS 
being associated with cardiovascular mortality, including vascular inflammation (11), platelet aggregation (12), oxidative stress (13), and coronary atherosclerosis (14). However, the association between baseline DHEAS level and cardiovascular or all-cause mortality has yielded conflicting findings in the general population (15). Differences in baseline age may partly contribute to the contradictory finding of prior studies.

To date, no previous meta-analysis has specially performed to evaluate the association of low blood DHEAS level with cardiovascular or all-cause mortality risk in the general population. DHEAS exhibits a steady decline with age, reaching less than $20 \%$ of its maximal value (16). Considering age is an obvious heterogeneity when analyzing the predictive role of DHEAS level, this meta-analysis specially focused on the association of serum/plasma DHEAS level with cardiovascular or all-cause mortality risk in the elderly population. We presented the following article in accordance with the PRISMA reporting checklist (available at http://dx.doi.org/10.21037/apm-20-441).

\section{Methods}

\section{Search strategy}

Relevant studies were identified by a comprehensive search of PubMed and Embase databases from their inceptions to 4 February, 2019. Literature search was performed using a combination of the following keywords without language restriction: "dehydroepiandrosterone sulfate" AND "mortality" OR "death". In addition, we also manually scanned the reference lists of pertinent articles for any possible missing studies.

\section{Study selection}

Inclusion criteria were the follows: (I) longitudinal observational studies that performed in the elderly population; (II) baseline serum/plasma DHEAS level as exposure; (III) outcome of interests were cardiovascular or all-cause mortality; (IV) provided multivariable-adjusted risk estimate of cardiovascular or all-cause mortality for categorical analysis of circulating DHEAS level. Studies were removed according to the following exclusion criteria: (I) participants not restricted in the elderly general population; (II) outcome measures were not of interest; (III) reporting risk estimate by continuous DHEAS level (per SD, per quartile or per log change in DHEAS); (IV) only provided unadjusted risk estimate of cardiovascular or all-cause mortality. Two authors independently assess the eligibility of the retrieved articles based on the above inclusion and exclusion criteria.

\section{Data extraction and quality assessment}

Data extraction and quality assessment were conducted by two independent authors. The following information were extracted from the eligible studies: surname of the first author, year of publication, geographic region, study design, sample sizes, proportion of women, mean age or age range at baseline, comparison of DHEAS category, number of endpoints, follow-up duration, fully adjusted risk ratio (RR), hazard ratio (HR), odds ratio (OR) with their corresponding $95 \%$ confidence intervals (CI), and adjustment for confounding factors. A 9-star NewcastleOttawa Scale (NOS) for cohort studies was applied to assess the methodological quality of the included studies (17). Studies awarding a score of $\geq 7$ stars were deemed as high-quality. Discrepancies in data extraction and quality assessment were resolved by discussion with a third author.

\section{Statistical analyses}

If the studies reported the lower DHEAS level as the reference category, we recalculated risk estimate taking the higher DHEAS as a reference group. HR and OR were directly deemed as the same measure of RR in this metaanalysis. Statistical heterogeneity across studies was checked using the Cochran's $\mathrm{Q}$ test (significance set at $\mathrm{P}<0.10$ ) and $\mathrm{I}^{2}$ statistic (significance set at $\mathrm{I}^{2} \geq 50 \%$ ). Meta-analyses were conducted using a random effect model in the presence of statistical heterogeneity or a fixed-effect model without statistical heterogeneity. Publication bias was scheduled for the Begg's rank correlation (18) and Egger's linear regression test (19). Subgroup analyses were planned by the sample sizes $(>1,000 v s .<1,000)$, geographic region (Europe $v s$. others), length of follow-up ( $>5 v s . \leq 5$ years), gender (women vs. men), and cutoff value of DHEAS level ( $>2$ categories $v s$. single). We performed leave-out one study sensitivity analysis at each turn to investigate the impact of individual studies on the overall pooled risk estimate. All statistical analyses were carried out using STATA 12.0 (Stata, College Station, TX, USA). 


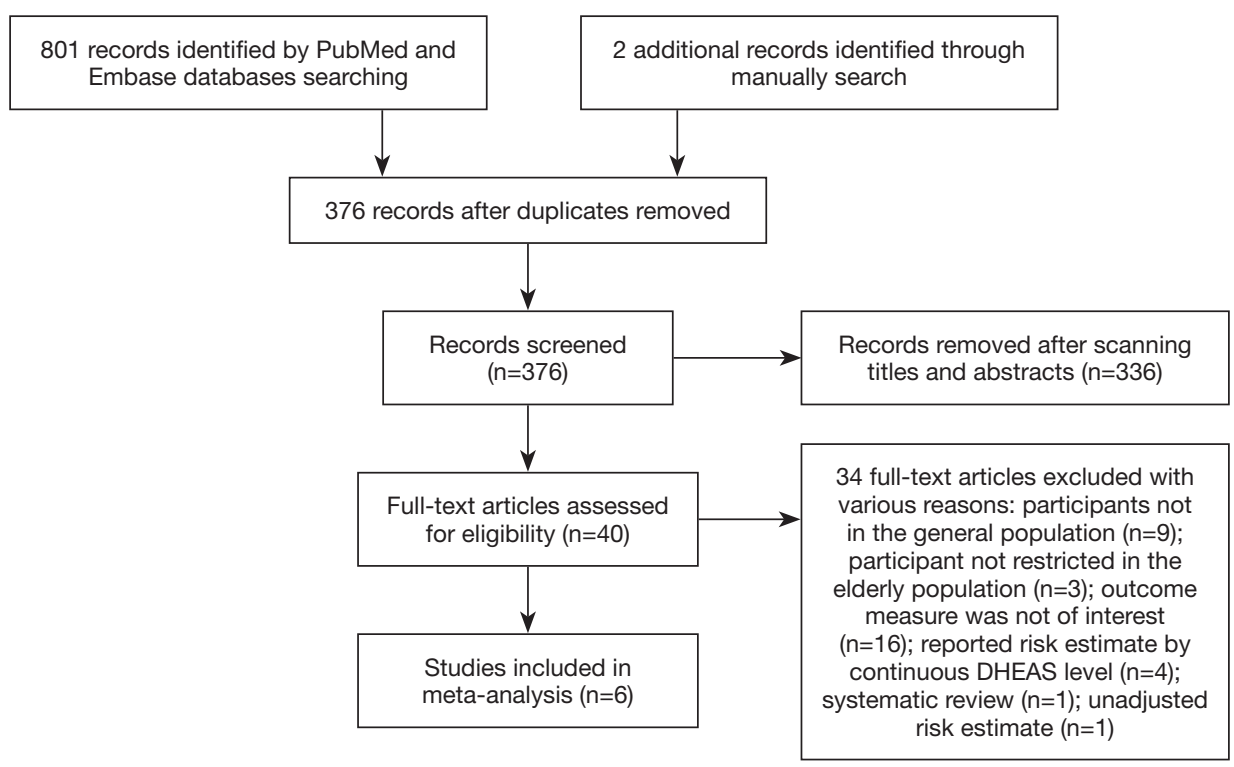

Figure 1 Flow diagram of study selection process.

\section{Results}

\section{Literature search and study characteristics}

Our initial literature search yielded 803 records, of which 376 were left after removal of duplicates. Of these, 40 fulltext potentially articles were assessed for eligibility and 6 studies (20-25) were finally included in this meta-analysis. A flowchart outlining our selection process is shown in Figure 1.

Table 1 summarizes the general characteristics of the included studies. In total, this meta-analysis included 6,744 participants, with 1,176 all-cause death and 282 cardiovascular death events. Sample size of participants varied from 214 to 2,644 individuals. The age of participants was 65 years or over. These included studies were published from 2001 to 2012. All the included studies were prospective cohort designs. Study populations came from the United Kingdom (20), Sweden (23), Italy $(22,25)$, United States (21), and Japan (24). The duration of followup ranged from 2.7 to 8.0 years. For methodological quality assessment, five studies (83.3\%) were graded as high quality.

\section{All-cause mortality}

All the included studies reported the association between circulating DHEAS level and risk of all-cause mortality. As shown in Figure 2, a fixed-effect model meta-analysis showed the pooled RR of all-cause mortality was 1.46 (95\% CI: $1.25-1.70)$ when compared the lowest to the reference higher category of DHEAS level. There was no significant heterogeneity between studies $\left(\mathrm{I}^{2}=41.6 \%\right.$; $\mathrm{P}=0.113)$. Publication bias was not observed based on the results of Begg's test $(\mathrm{P}=1.000)$ and Egger's test $(\mathrm{P}=0.489)$. Sequential exclusion of any single study minimally altered the overall pooled risk estimate (RR range: 1.41-1.58). Stratified analyses indicated that the significant association of low serum DHEAS level with all-cause mortality risk was consistently found in each subgroup except for the single cutoff DHEAS comparison subgroup (Table 2).

\section{Gender-specific all-cause mortality}

Two studies $(22,23)$ included the men and two studies $(21,24)$ enrolled the women only. Two studies $(20,25)$ recruited the women and men separately. As shown in Figure 3, when the lowest serum DHEAS level were compared with the reference higher DHEAS category, the pooled RR of allcause mortality was 1.41 (95\% CI: $1.18-1.69 ; \mathrm{I}^{2}=0.0 \%$, $\mathrm{P}=0.860$ ) for men and 1.72 (95\% CI: $0.99-2.99 ; \mathrm{I}^{2}=70.5 \%$, $\mathrm{P}=0.017)$ for women in a random-effect model.

\section{Cardiovascular mortality}

Two studies $(20,25)$ reported the association between circulating DHEAS level and risk of cardiovascular mortality. As shown in Figure 4, a fixed-effect model metaanalysis indicated the pooled RR of cardiovascular mortality 


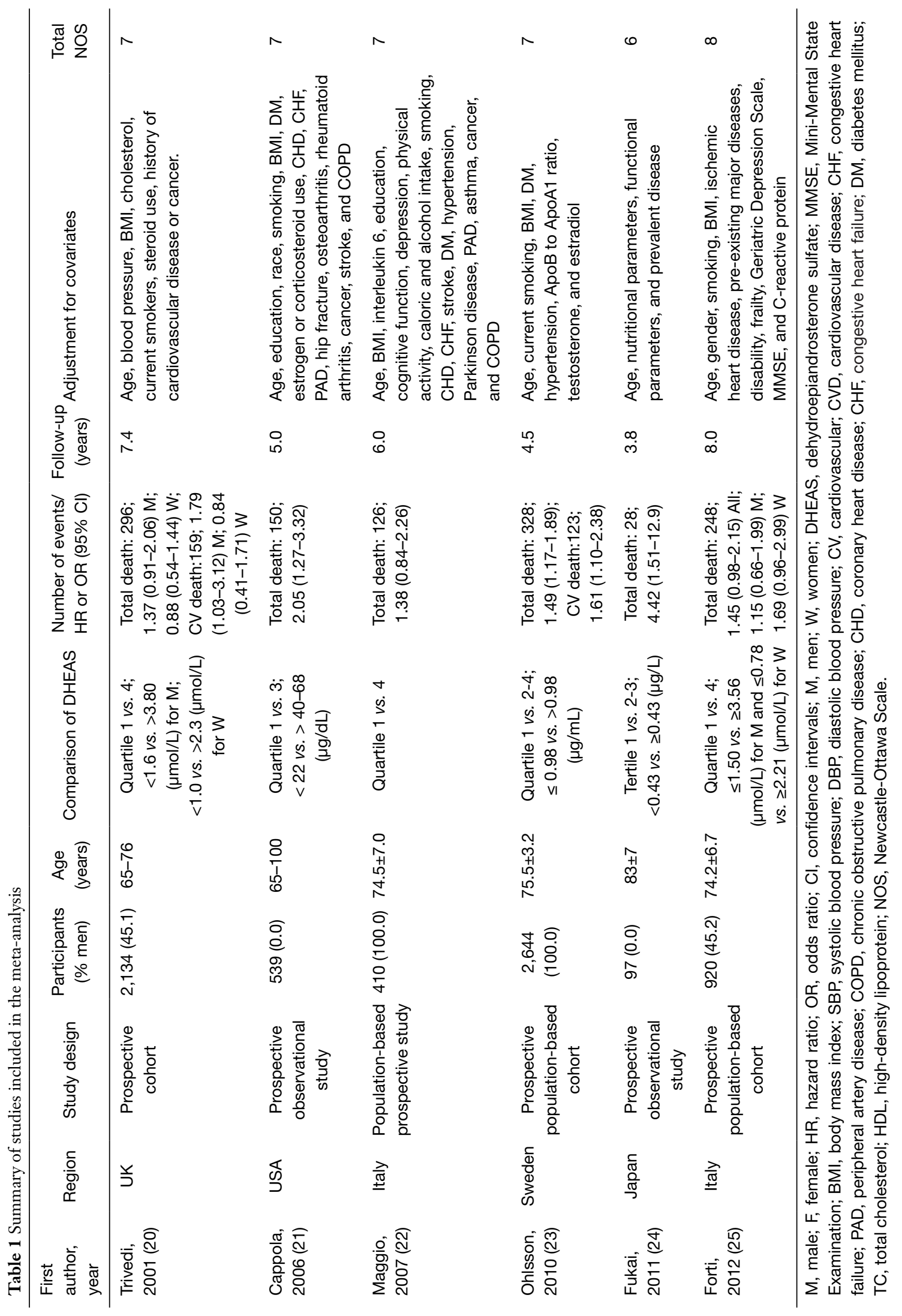




$$
\text { Study }
$$

ID
$\%$

RR $(95 \% \mathrm{Cl}) \quad$ Weight

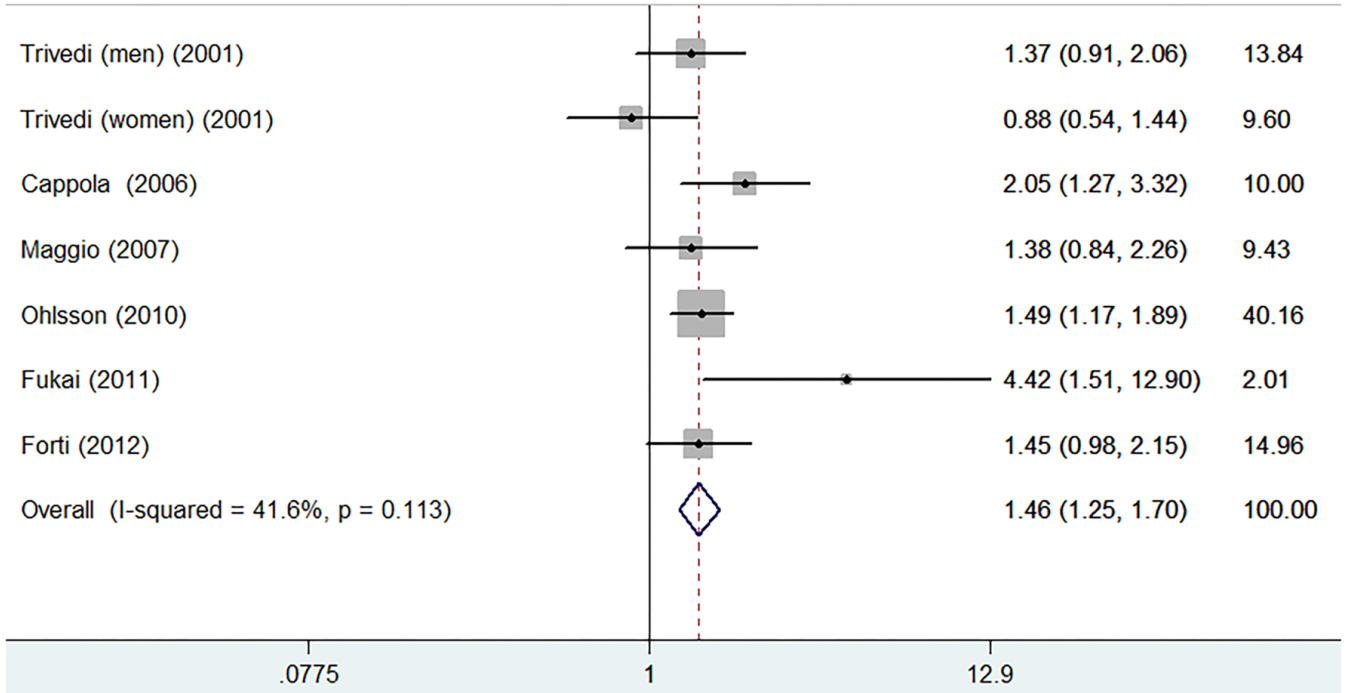

Figure 2 Forest plots showing pooled RR with $95 \%$ CI of all-cause mortality for the lower versus higher category of dehydroepiandrosterone sulfate level.

Table 2 Subgroup analyses of all-cause mortality

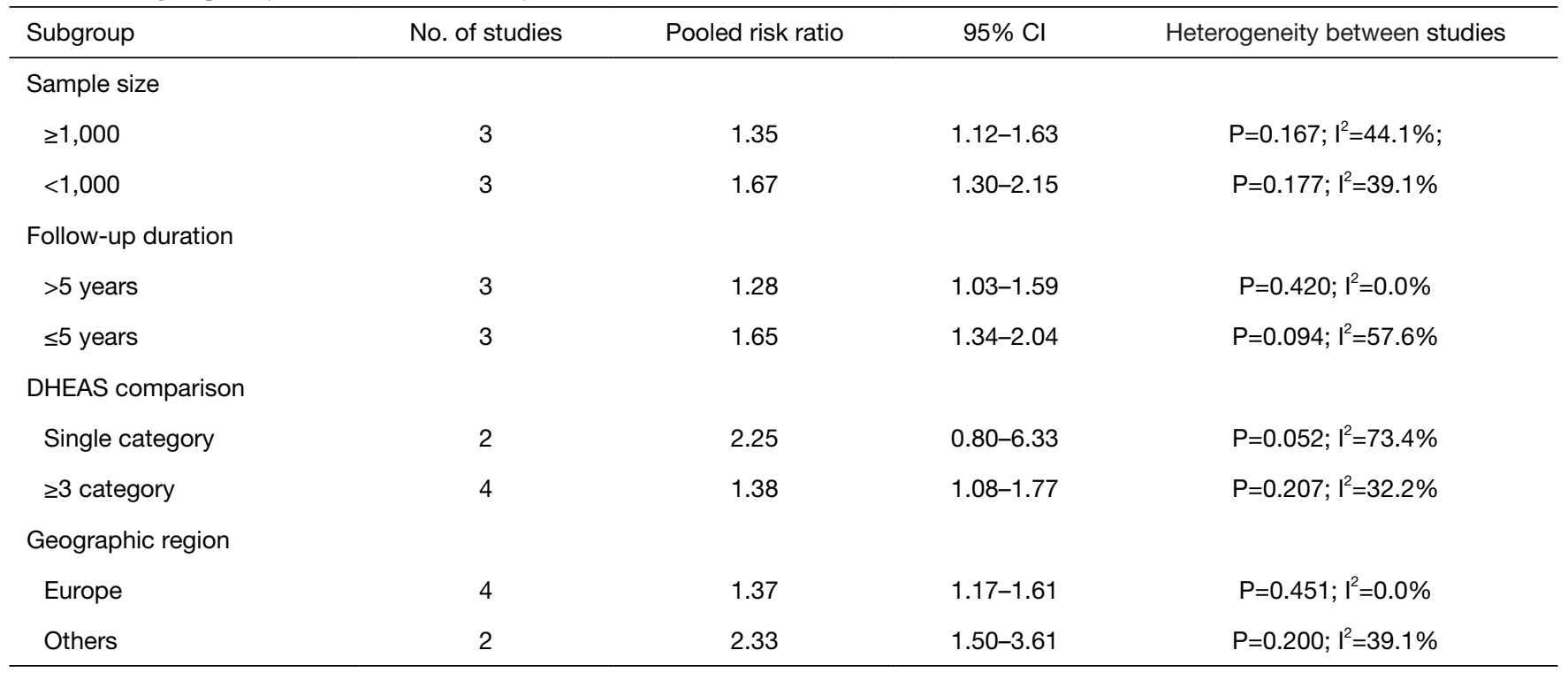




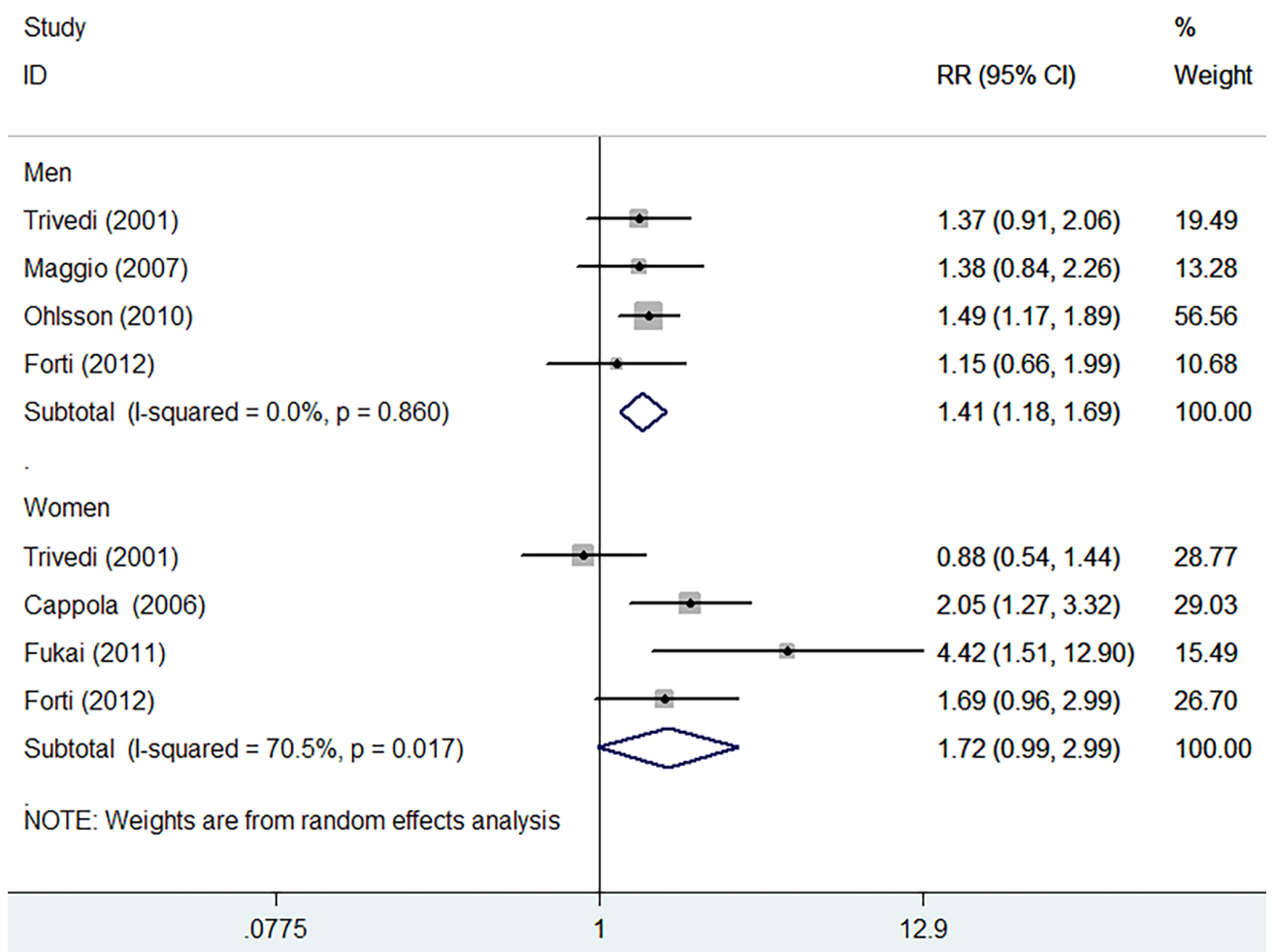

Figure 3 Forest plots showing pooled RR with $95 \%$ CI of all-cause mortality by gender.

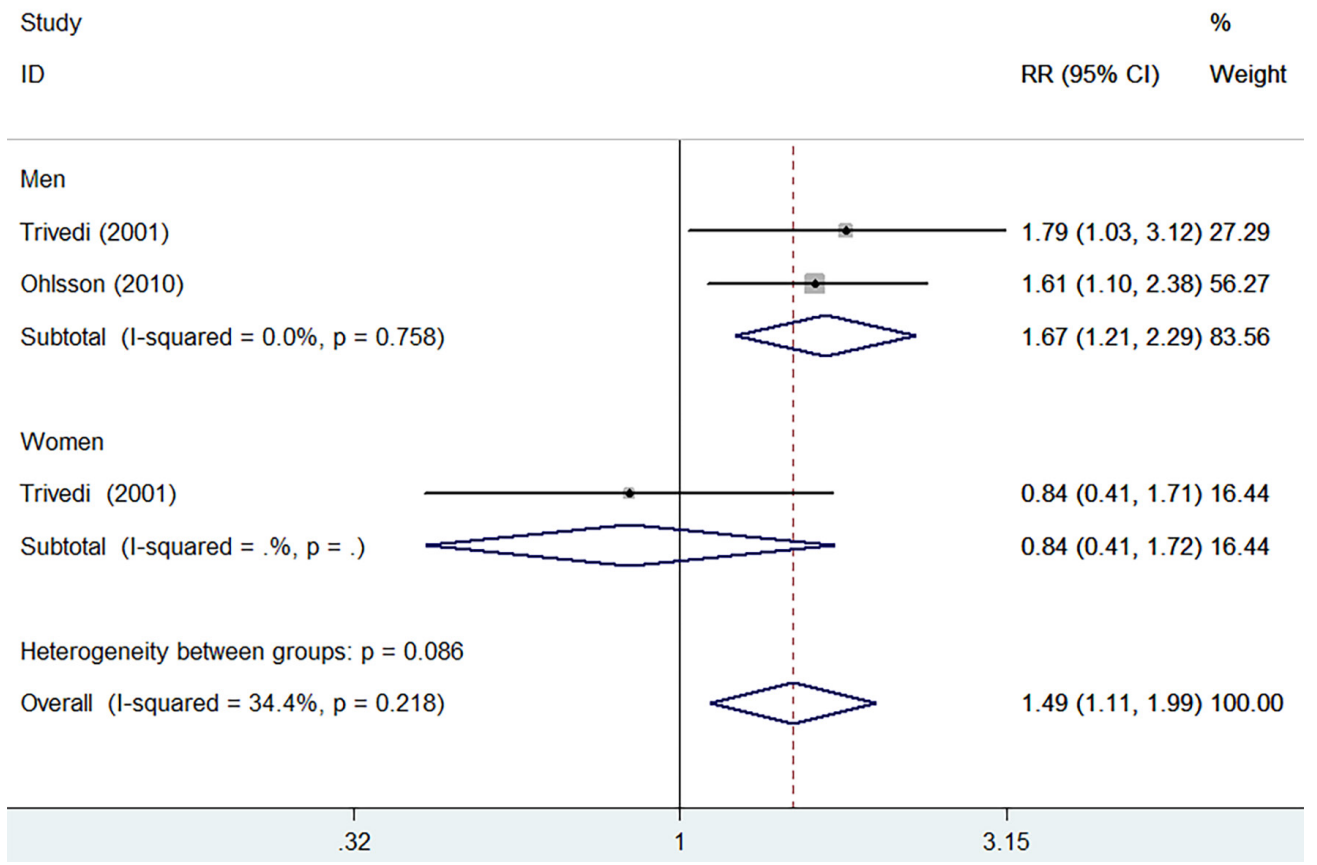

Figure 4 Forest plots showing pooled RR with 95\% CI of cardiovascular mortality for the lower versus higher category of dehydroepiandrosterone sulfate level. 
was 1.49 (95\% CI: 1.11-1.99) when compared the lowest to the reference higher category of DHEAS level. There was no evidence of significant heterogeneity between studies $\left(\mathrm{I}^{2}=34.4 \% ; \mathrm{P}=0.218\right)$. Subgroup analyses by gender indicated that the risk cardiovascular mortality was significant in men (RR 1.67; 95\% CI: 1.21-2.29) but not in women (RR 0.84; 95\% CI: 0.41-1.72).

\section{Discussion}

This meta-analysis suggests that low circulating DHEAS level is associated with an increased risk of all-cause mortality in the elderly population. In elderly men (over 65 years) of the general population, individuals with the lowest circulating DHEAS level had a $46 \%$ higher risk of all-cause mortality. Elderly men with the lowest circulating DHEAS level exhibited a $67 \%$ higher risk of cardiovascular mortality. However, low circulating DHEAS level appeared to have no clear effects on cardiovascular or all-cause mortality in elderly women.

In accordance with our study, a previous systematic review and meta-analysis (26) suggested that cardiovascular disease patients with lower DHEAS levels had a $47 \%$ and $58 \%$ higher risk of all-cause mortality and fatal cardiovascular event. However, this meta-analysis included heterogeneous populations but not focused on the elderly population. To our knowledge, the present study is the first meta-analysis to summarize the association between circulating DHEAS level and cardiovascular or allcause mortality in elderly general population. Apart from DHEAS, low endogenous testosterone level was associated with higher risk of all-cause and cardiovascular death in community-based studies of men (27). Aging process is associated with a decline in anabolic hormones (28). Therefore, multiple deficiencies in contamination of testosterone and DHEAS may have synergistic effects in predicting mortality in older men.

In our meta-analysis, gender-specific analysis of all-cause mortality indicated that the association of low DHEAS level with all-cause mortality risk was only observed in men but not in women. Analysis with cardiovascular mortality also yielded similar findings. These results suggest that DHEAS may exhibit different physiological effects among men and women. Also, directions of the pooling risk estimate for the DHEAS level with all-cause and cardiovascular mortality in women were different. This finding may be partly explained by the differences in the predominance of other sex hormones (29). Dehydroepiandrosterone can exert either an estrogen-like or androgen-like effect. Its metabolism to testosterone may increase the cardiovascular disease in postmenopausal women (30). A U-shaped association between DHEAS level and cardiovascular mortality was observed in postmenopausal women (31) and either low or high DHEAS level was at increased risk. High estrogen level may counterbalance the androgenic effects of dehydroepiandrosterone in the premenopausal women (32).

The mechanisms underlying the association of low DHEAS with mortality remain poorly elucidated. There may be several mechanisms by which endogenous DHEAS affects mortality in elderly men: (I) given both low serum testosterone and estradiol can predict mortality in elderly men, DHEAS can locally convert to testosterone and/or estradiol in target tissues; (II) DHEAS as its precursor has been implicated in antiinflammatory and immune regulatory activity (33).

Several limitations of the current meta-analysis should be mentioned. First, a single baseline determination of DHEAS level might have led to overestimate or underestimate the relationship of DHEAS level with mortality. Second, different cutoff values of circulating DHEAS level reported in the analyzed studies prevent us to recommend the specific threshold of low DHEAS level in clinical settings. Third, the validity of our findings was restricted to the elderly general population, which may not generalize to younger persons. Finally, a U-shaped association between DHEAS level and mortality has been reported (21). Thus, use of the highest DHEAS level as reference may have led to underestimate the association between the lowest DHEAS level and mortality.

\section{Conclusions}

In conclusion, this meta-analysis indicates that low circulating DHEAS level is associated with increased risk of all-cause mortality in the elderly population. However, future randomized controlled trials investigating the effect of DHEA therapy on individuals with DHEAS deficiency are warranted before DHEAS supplementation for longevity can be recommended.

\section{Acknowledgments}

Funding: None.

\section{Footnote}

Reporting Checklist: The authors have completed the 
PRISMA reporting checklist. Available at http://dx.doi. org/10.21037/apm-20-441

Conflicts of Interest: All authors have completed the ICMJE uniform disclosure form (available at http://dx.doi. org/10.21037/apm-20-441). The authors have no conflicts of interest to declare.

Ethical Statement: The authors are accountable for all aspects of the work in ensuring that questions related to the accuracy or integrity of any part of the work are appropriately investigated and resolved.

Open Access Statement: This is an Open Access article distributed in accordance with the Creative Commons Attribution-NonCommercial-NoDerivs 4.0 International License (CC BY-NC-ND 4.0), which permits the noncommercial replication and distribution of the article with the strict proviso that no changes or edits are made and the original work is properly cited (including links to both the formal publication through the relevant DOI and the license). See: https://creativecommons.org/licenses/by-nc-nd/4.0/.

\section{References}

1. Traish AM, Kang HP, Saad F, et al. Dehydroepiandrosterone (DHEA)--a precursor steroid or an active hormone in human physiology. J Sex Med 2011;8:2960-82;quiz 2983.

2. Prough RA, Clark BJ, Klinge CM. Novel mechanisms for DHEA action. J Mol Endocrinol 2016;56:R139-55.

3. Labrie F, Luu-The V, Belanger A, et al. Is dehydroepiandrosterone a hormone? J Endocrinol 2005;187:169-96.

4. Orentreich N, Brind JL, Rizer RL, et al. Age changes and sex differences in serum dehydroepiandrosterone sulfate concentrations throughout adulthood. J Clin Endocrinol Metab 1984;59:551-5.

5. Vermeulen A. Dehydroepiandrosterone sulfate and aging. Ann N Y Acad Sci 1995;774:121-7.

6. Enomoto M, Adachi H, Fukami A, et al. Serum dehydroepiandrosterone sulfate levels predict longevity in men: 27-year follow-up study in a communitybased cohort (Tanushimaru study). J Am Geriatr Soc 2008;56:994-8.

7. Guder G, Frantz S, Bauersachs J, et al. Low circulating androgens and mortality risk in heart failure. Heart 2010;96:504-9.
8. Jansson JH, Nilsson TK, Johnson O. von Willebrand factor, tissue plasminogen activator, and dehydroepiandrosterone sulphate predict cardiovascular death in a 10 year follow up of survivors of acute myocardial infarction. Heart 1998;80:334-7.

9. Hsu HJ, Yen CH, Chen CK, et al. Low plasma DHEA-S increases mortality risk among male hemodialysis patients. Exp Gerontol 2012;47:950-7.

10. Zurfluh S, Nickler M, Ottiger M, et al. Association of adrenal hormone metabolites and mortality over a 6-year follow-up in COPD patients with acute exacerbation. Clin Chem Lab Med 2018;56:669-80.

11. Altman R, Motton DD, Kota RS, et al. Inhibition of vascular inflammation by dehydroepiandrosterone sulfate in human aortic endothelial cells: roles of PPARalpha and NF-kappaB. Vascul Pharmacol 2008;48:76-84.

12. Bertoni A, Rastoldo A, Sarasso C, et al. Dehydroepiandrosterone-sulfate inhibits thrombininduced platelet aggregation. Steroids 2012;77:260-8.

13. Goy B, Atmaca M, Aslan M, et al. Relationship between serum DHEAS and oxidative stress levels of body mass index in healthy postmenopausal women. Redox Rep 2016;21:61-6.

14. Herrington DM, Gordon GB, Achuff SC, et al. Plasma dehydroepiandrosterone and dehydroepiandrosterone sulfate in patients undergoing diagnostic coronary angiography. J Am Coll Cardiol 1990;16:862-70.

15. Ohlsson C, Vandenput L, Tivesten A. DHEA and mortality: what is the nature of the association? J Steroid Biochem Mol Biol 2015;145:248-53.

16. Dharia S, Parker CR, Jr. Adrenal androgens and aging. Semin Reprod Med 2004;22:361-8.

17. Wells G, Shea B, O'Connell D, et al. The NewcastleOttawa Scale (NOS) for assessing the quality if nonrandomized studies in meta-analyses. Available online: http://www.ohri.ca/programs/clinical_epidemiology/ oxford.asp

18. Begg CB, Mazumdar M. Operating characteristics of a rank correlation test for publication bias. Biometrics 1994;50:1088-101.

19. Egger M, Davey Smith G, Schneider M, et al. Bias in meta-analysis detected by a simple, graphical test. BMJ 1997;315:629-34.

20. Trivedi DP, Khaw KT. Dehydroepiandrosterone sulfate and mortality in elderly men and women. J Clin Endocrinol Metab 2001;86:4171-7.

21. Cappola AR, Xue QL, Walston JD, et al. DHEAS levels and mortality in disabled older women: the Women's 
Health and Aging Study I. J Gerontol A Biol Sci Med Sci 2006;61:957-62.

22. Maggio M, Lauretani F, Ceda GP, et al. Relationship between low levels of anabolic hormones and 6-year mortality in older men: the aging in the Chianti Area (InCHIANTI) study. Arch Intern Med 2007;167:2249-54.

23. Ohlsson C, Labrie F, Barrett-Connor E, et al. Low serum levels of dehydroepiandrosterone sulfate predict all-cause and cardiovascular mortality in elderly Swedish men. J Clin Endocrinol Metab 2010;95:4406-14.

24. Fukai S, Akishita M, Yamada S, et al. Plasma sex hormone levels and mortality in disabled older men and women. Geriatr Gerontol Int 2011;11:196-203.

25. Forti P, Maltoni B, Olivelli V, et al. Serum dehydroepiandrosterone sulfate and adverse health outcomes in older men and women. Rejuvenation Res 2012;15:349-58.

26. Wu TT, Chen Y, Zhou Y, et al. Prognostic Value of Dehydroepiandrosterone Sulfate for Patients With Cardiovascular Disease: A Systematic Review and MetaAnalysis. J Am Heart Assoc 2017;6:e004896.

Cite this article as: Li R, E L, Zha N. Circulating dehydroepiandrosterone sulfate level and cardiovascular or allcause mortality in the elderly population: a meta-analysis. Ann Palliat Med 2020;9(5):3537-3545. doi: 10.21037/apm-20-441
27. Araujo AB, Dixon JM, Suarez EA, et al. Clinical review: Endogenous testosterone and mortality in men: a systematic review and meta-analysis. J Clin Endocrinol Metab 2011;96:3007-19.

28. Lamberts SW, van den Beld AW, van der Lely AJ. The endocrinology of aging. Science 1997;278:419-24.

29. Barrett-Connor E, Goodman-Gruen D. The epidemiology of DHEAS and cardiovascular disease. Ann N Y Acad Sci 1995;774:259-70.

30. Armeni E, Lambrinoudaki I. Androgens and cardiovascular disease in women and men. Maturitas 2017;104:54-72.

31. Barrett-Connor E, Khaw KT. Absence of an inverse relation of dehydroepiandrosterone sulfate with cardiovascular mortality in postmenopausal women. $\mathrm{N}$ Engl J Med 1987;317:711.

32. Ebeling P, Koivisto VA. Physiological importance of dehydroepiandrosterone. Lancet 1994;343:1479-81.

33. Dillon JS. Dehydroepiandrosterone, dehydroepiandrosterone sulfate and related steroids: their role in inflammatory, allergic and immunological disorders. Curr Drug Targets Inflamm Allergy 2005;4:377-85. 